\title{
The prevalence and severity of syndrome $Z$ in severely obese female patients on the waiting list for bariatric surgery. A cross-sectional study protocol
}

\author{
Eduardo A. Perez1', Wilson Rodrigues Junior ${ }^{1}$, Anderson Soares Silva², Jessica Julioti Urbano², Carina Lottenberg'1, \\ Ana Carolina Monteiro Brunetti², Vera Lucia dos Santos Alves' ${ }^{1}$, Patrícia Clemente Oliveira², \\ Roger André Oliveira Peixoto², Ezequiel Fernandes Oliveira², Sergio Roberto Nacif2,3, Israel Reis dos Santos², \\ Elias Jirjoss Ilias', Carlos Alberto Malheiros ${ }^{1}$, Luis V F Oliveira², Roberto Stirbulov ${ }^{1}$
}

\begin{abstract}
Introduction: The Syndrome Z (SyndZ) has been drawing scientific community's attention on the past years. It is characterized by the interaction between obstructive sleep apnea (OSA) and Metabolic Syndrome (MetS). On its turn, MetS is knew for the association with various metabolic disturbances occurring simultaneously in the same patient, those of which include central obesity, systemic hypertension (SH), glucose intolerance or insulin resitance, low serum high-density lipoprotein cholesterol (HDL), and elevated serum triglycerides. OSA is a prevalent condition all over the world, and presents potential adverse health outcomes, with a particular impact in cardiovascular diseases. Therefore, OSA and MetS present important cardiovascular risk factors, that when superimposed can mean a higher cardiovascular morbi-mortality. This associated pathogenesis has not been completely elucidated. Objectives: A pilot study to determine the prevalence and severity of SyndZ in severely obese female patients on the waiting list for bariatric surgery. Methods/Design: This cross-sectional study will be conducted with severely obese female patients, on waiting list for bariatric surgery, from the Santa Casa de Misericordia de São Paulo Hospital, in the city of Sao Paulo (Brazil). Demographic, anthropometric and clinical data will be collected, as well as blood's biochemical analysis. The patients will also undergo standard overnight polysomnography at the Sleep Laboratory of Nove de Julho University.
\end{abstract}

Keywords: Syndrome Z; Metabolic syndrome; Obstructive sleep apnoea; Severe obesity; Polysomnography.

Trial Registration: This protocol follows the STROBE (STrengthening the Reporting of OBservational studies in Epidemiology) and the study has been registered on ClinicalTrials.gov NCT02409160.

\section{BACKGROUND}

The worldwide prevalence of obesity has more than doubled from 1980 to 2014. In 2014, more than 1.9 billion adults, 18 years and older, were overweight, meaning $39 \%$ of the population. Therefore, $38 \%$ of the male population and $40 \%$ of the female population worldwide were overweight. Overall, about $13 \%$ of the world's adult population ( $11 \%$ of men and $15 \%$ of women) were obese in $2014 .{ }^{1}$

Most of the world's population lives in countries where overweight and obesity kills more people than those who are underweight. Also, 41 million children under the age of 5 years old were overweight or obese in 2014. ${ }^{1}$ Contextualizing, overweight and obesity are defined as abnormal or excessive fat accumulation that presents a risk to health. A crude population measure of obesity is the body mass index (BMI), a person's weight (kilograms) divided by the square of his or her height (meters). A person with a BMI equal to or more than 25 is considered overweight, and with a BMI of 30 or more is generally considered obese.

Overweight and obesity are major risk factors for a number of chronic diseases, including diabetes, cardiovascular diseases and cancer. Once considered a problem only in high income countries, overweight and obesity are now dramatically on the rise in low- and middle-income countries, particularly in urban settings. ${ }^{2}$ In the United Kingdom, prevalence of obesity amongst people over 16 years old is $24,8 \%$ and the prevalence of severe obesity is approximately $2,5 \%$ (3,2\% for women 
and $1,7 \%$ for men), meanwhile $6,6 \%$ of American adults are severely obese. ${ }^{2-5}$ At $B M I \geq 40 \mathrm{~kg} / \mathrm{m}^{2}$, obesity is considered a dangerous dysfunction, generally associated with many diseases. ${ }^{5}$ Amongst the most frequent comorbities associated to obesity are systemic hypertension $(\mathrm{SH})^{6-7}$ and diabetes mellitus type II (DM) ${ }^{8}$ The epidemic of obesity results in major health related consequences in different ethnic populations. There is increasing awareness of the role of obesity in the development of cardiometabolic disease in recent years. ${ }^{9}$

The metabolic syndrome (MetS) is strongly linked to obesity, and is considered a constellation of interrelated risk factors of metabolic origin that are accompanied by increased risk for cardiovascular disease and DM. This collection of unhealthy body measurements and abnormal laboratory test results include atherogenic dyslipidemia, $\mathrm{SH}$, glucose intolerance, proinflammatory state, and a prothrombotic state. ${ }^{10,11}$

In 1988, Reaven described "a cluster of risk factors for diabetes and cardiovascular disease" and named it "Syndrome $X$ ". His main contribution was an introduction of the concept of insulin resistance. MetS is defined by a constellation of interconnected physiological, biochemical, clinical, and metabolic components that directly increases the risk of atherosclerotic cardiovascular disease, DM, and many others causes of mortality. ${ }^{10,11}$ Over the years the name "Syndrome X" was substituted by "Metabolic Syndrome" and recently it became known as "Cardiometabolic Syndrome". ${ }^{12-14}$ In the United States, age-adjusted MetS prevalence among adult population was estimated to be $24-25 \%$. Similarly, the prevalence of MetS in 7 European countries was approximately $23 \%$. And, it was estimated that $20 \%-25 \%$ of South Asians have developed MetS. ${ }^{15-16}$

A widely used definition of this syndrome was originally released on the Third Report of the National Cholesterol Education Program Expert Panel on Detection, Evaluation and Treatment of High Blood Cholesterol in Adults (Adult Treatment Panel III [ATP III]) in 2000. ${ }^{17}$ According to ATP III the diagnosis of MetS is based on three out of five simple clinical measures: increased waist circumference (abdominal obesity), elevated triglycerides, reduced low serum high-density lipoprotein cholesterol (HDL), elevated blood pressure, and elevated glucose. ${ }^{10}$

Obstructive Sleep Apnea (OSA) is a common clinical condition present in MetS patients, which was confirmed by recent studies that noted a prevalence higher than $60 \%$ for this association. ${ }^{18,19}$ Some observational studies have shown that OSA and MetS exhibit similar pathophysiologic substrates for cardiovascular disease (CVD), being the arterial pressure's increase a common consequence for these pathologies. Which raises a discussion of wether MetS and OSA could have an additive effect on cardiovascular risks. There already is considerable scientific evidence, based on studies on humans and animals, suggesting that OSA can influence all
MetS' clinical aspects, including obesity ${ }^{20}$, insulin resistance ${ }^{21}$ and dyslipidemia. ${ }^{22,23}$ Other studies also have shown the coexistence between these two pathologies. ${ }^{24,26}$

The most important risk factor for OSA is obesity ${ }^{27}$, mainly due to the fat buildup in the high portion of the abdomen and cervical region. Approximately, 60 to $70 \%$ of patients that present OSA are obese. In addition, its incidence amongst patients that present degree 3 obesity is 12 to 30 times higher than in general population. ${ }^{28}$

OSA is characterized by recurrent partial or complete upper airway obstruction episodes during sleep in the presence of respiratory effort, with consequent drop of oxyhemoglobin's saturation, leading to negative intrathoracic pressures and awakenings. ${ }^{29}$ The gold standard for OSA's diagnosis is the basal nighttime polyssomnography (PSG), characterized by the simultaneous registering of physiological parameters during a night's sleep, analyzing its stages, the respiratory pattern, cardiovascular function and body movements. ${ }^{29,30}$

Aside from the possibility that OSA identifies a group of patients with a cluster of vascular risk factors, there may be specific effects of OSA which further increase the cardiovascular consequences of this possible risk factor association. Thus, "syndrome X" may actually include OSA and could be better considered as "syndrome Z" (SyndZ). ${ }^{31}$

\section{AIMS AND HYPOTHESIS}

\section{Study objectives}

\section{Primary objective}

To determine the prevalence and severity of SyndZ in severely obese female patients on the waiting list for bariatric surgery, and whether the presence of SyndZ is correlated with age, BMI, and neck circumference.

\section{Secondary objectives}

To identify the presence of sleep disorders through the PSG in severely obese female patients on the waiting list for bariatric surgery. Our hypothesis is that there should be a correlation between the severity of Apnea/Hypopnea index $(\mathrm{AHI})$ and the physiological and clinical variables from the patients involved in this study.

\section{Methods and design}

\section{Study design and setting}

A consecutive single-center cross-sectional study will be performed, to investigate the prevalence and severity of SyndZ in severely obese women. This study will be conducted in the Medical School of Santa Casa de Sao Paulo and at the Sleep Laboratory of the Nove de Julho University (UNINOVE). The design and conduct of this study followed the guidelines 
of the Strengthening the Reporting of Observational Studies in Epidemiology (STROBE) statement, according to figure 1.

\section{Ethical and legal aspects}

The study will be conducted in accordance with the ethical standards established in 1961 by Declaration of Helsinki (revised Hong Kong in 1989 and Edinburgh, Scotland, in 2000) and will be in accordance with the Regulatory Guidelines and Standards of Research Involving Human Subjects of Comissao Nacional de Etica em Pesquisa (CONEP) do Ministerio da Saude published in December, 2012. This protocol study has been approved by the Research Ethics Committee of the Nove de
Julho University (Brazil), process no. 360.488 and is registered in the ClinicalTrials.gov no. NCT02409173. The signing of the free and informed consent will be required from all patients, and they will be allowed to withdraw from the study at any time without any negative consequences. Patients with severe clinical problems will be referred to the appropriate treatment aimed at their health status and quality of life.

\section{Subjects and recruitment procedure}

Female patients, with severe obesity on waiting list for bariatric surgery, according to the identification criteria (BMI between $40 \mathrm{~kg} / \mathrm{m}^{2}$ and $50 \mathrm{~kg} / \mathrm{m}^{2}$ or BMI between

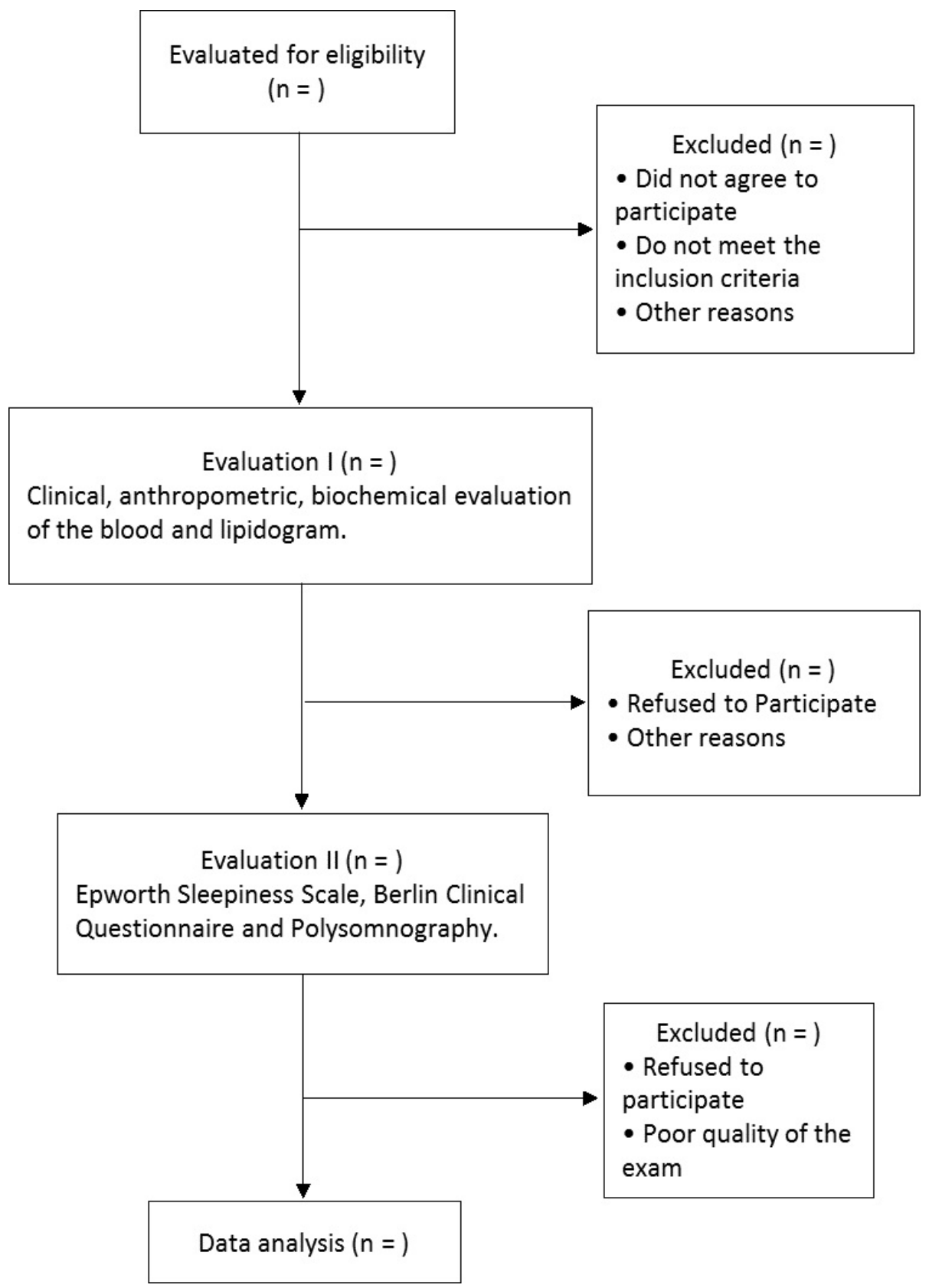

Figure 1. Flow diagram of the study. 
$35 \mathrm{~kg} / \mathrm{m}^{2}$ and $39,9 \mathrm{~kg} / \mathrm{m}^{2}$ associated with comorbities) will be recruited from the surgery unit of the Santa Casa de Misericordia from the city of Sao Paulo (Brazil). The participants will be recruited consecutively and selected according to the eligibility criteria from standardized protocol. The eligibility criteria are as follow.

\section{Eligibility criteria}

The following inclusion criteria must be fulfilled at the start of the study: female patients, age of 18-65 years, stage III severe obesity (BMI of $\geq 40 \mathrm{~kg} / \mathrm{m} 2$ or $\geq 35 \mathrm{~kg} / \mathrm{m} 2$ with comorbities), awaiting BS, a documented history of failed conventional weight loss attempts, and the ability to understand and agree to participate in the study, based on a signed informed consent. The exclusion criteria will be $\mathrm{BMI}$ of $>55 \mathrm{~kg} / \mathrm{m2}$, alcohol or drug abuse, cancer, and any other cardiorespiratory and/or medical condition that contraindicates participation in the study.

\section{Clinical evaluation}

The clinical evaluation of the patients included on this study will happen before bariatric surgery is held. All subjects will be submitted to the same evaluation protocol described subsequently. Evaluation will be done by a doctor and a physiotherapist, following standard recommended procedures and using precise instruments $\mathrm{s}^{32}$, including body weight $(\mathrm{kg})$, height $(\mathrm{m}), \mathrm{BMI}$, neck, hip and waist circumference $(\mathrm{cm})^{33}$, respiratory and cardiac rates, arterial pressure, specific questionnaires for OSA and excessive daytime sleepiness (EDS) and a quality of life questionnaire. Some relevant clinical information will also be collected from patient's charts at the occasion of the first appointment.

Blood pressure will be measured between the hours of 8 and 11 a.m. after the patient will have rested in a sitting position for 5 minutes, using the sphygomanometer (Omron automatic oscillometric digital blood pressure monitor, model HEM-705CP, Omron Corporation, Tokyo, Japan) to obtain the systolic blood pressure (SBP) and diastolic blood pressure (DBP). The measurements will be obtained three times, and the average values will be used in the data analysis. The time in between measurements will be one minute.

\section{Laboratorial analysis of blood's biochemistry}

The analyses will be done through blood samples collection for the execution of complete hemogram, sodium and potassium dosage, glycaemia, creatinine, triglycerides, HDL cholesterol and total cholesterol. All samples will be collected in a laboratory of biochemical analyzes at hospital by a qualified technician and using disposable material, being followed the bio-security standards.

\section{Fasting glucose}

Fasting glucose will be measured after an overnight fast in whole blood using a glucose-oxidase-based assay (YSI 2300, Analytical Technologies, Farnborough, UK).

\section{Insulin resistance}

Insulin will be quantified using the IMMULITE 2000 Insulin assay, a solid-phase, two-site, chemiluminescent enzyme-labelled immunometric assay, and the Immulite 2000 automated analyser (Diagnostic Products Corporation, Los Angeles, CA, USA). Insulin resistance will be assessed from fasting glucose and insulin values using homeostasis model assessment (HOMA) calculations, previously validated against the hyperinsulinaemic euglycaemic clamp.

Lipids

Fasting cholesterol, triglyceride (Bayer Corporation, Tarrytown, NY, USA), and HDL cholesterol (Sigma Diagnostics, St. Louis, MO, USA) concentrations will be measured after an overnight fast using an immunocolourimetric assay on an ADVIA ${ }^{\circledR}$ 1650 chemistry system (Bayer Corporation, Tarrytown, NY, USA). Low-densitylipoprotein (LDL) cholesterol will be derived using the Friedwald equation.

\section{Waist and neck circumferences}

Measurements from neck and waist circumferences will be done with the use of a metric tape of seven $\mathrm{mm}$ width. The measuring points will be standardized as following, according to scientific literature. For the waist's circumference, the measuring in the middle between the inferior border of the last rib and the iliac crest will be used, fixating the tape's zero point to the thorax from the axillary line's level which is going to be used (axillary, xiphoidian or abdominal). The neck's circumference will be taken horizontally on the cricoid cartillage. ${ }^{34,35}$

\section{Epworth Sleepiness Scale}

The Epworth Sleep Scale (ESS) is a self applicable questionnaire, based in questions about situations of excessive sleepiness envolving daily activities. The subjects will be oriented to classify in a scale of 0 to 3 the probability of feeling sleepy or falling asleep in eight different sittuations. Being zero no probability of falling asleep at all, one small probability of it happening, two moderate probability and three high probability. ${ }^{36,37}$

\section{Berlin Questionnaire}

The Berlin Questionnaire (BQ) is a clinical questionnaire of acknowledged efficacy on verifying risk for OSA. This instrumment is composed by ten items, organized in three categories relative to snore and OSA (containing 5 items), daytime sleepiness (4 items) SH and obesity (1 item). Any bolded answer is considered positive. The punctuation is divided in two categories in which category 1 is considered positive with two or more positive answers for questions 1 to 5 . Category 2 is considered 
positive with two or more positive answers to questions 6 to 8 and category 3 is considered positive if the answer for question 9 is yes or the $\mathrm{BMI}$ is over $30 \mathrm{~kg} / \mathrm{m}^{2}$. Two or more positive categories indicate a high risk for OSA. ${ }^{38}$

\section{Polysomnography}

PSG will be performed using a digital system Alice 5 (version 3.1.2 Flaga, Hs. Medical Devices, Islândia), at the Nove de Julho University Sleep Laboratory. All recording sensors will be fixed to the patient in a non-invasive manner using tape or elastic bands. The following physiological variables will be monitored simultaneously and continuously: four channels for the electroencephalogram (EEG) (C3-A2, C4-A1, O1-A2, O2-A1), two channels for the electrooculogram, four channels for the surface electromyogram (muscle of the submentonian region, and anterior tibialis muscle), one channel for an electrocardiogram (derivation V1 modified), airflow detection via two channels through a thermocouple and nasal pressure cannula, respiratory effort of the thorax and of the abdomen via x-trace belts, snoring, and body position via Alice 5 sensors, and arterial oxygen saturation and heart rate (HR) via an Alice 5 oximeter. All PSG exams will be performed and sleep stages visually scored according to standardized criteria for investigating sleep disorders. ${ }^{39,40} \mathrm{EEG}$ arousals, sleep-related respiratory events, and leg movements will be scored in accordance with the criteria established by the American Academy of Sleep Medicine Manual for Scoring Sleep and Associated Events. ${ }^{41}$

\section{Statistical analysis}

Normally distributed data will be presented as means \pm standard error of the mean, logarithmically transformed data as the geometric mean (95\% confidence interval for the mean), skewed data as the median (interquartile range), and categorical data as the number (percentage). Demographic data will be compared using the $95 \%$ confidence interval for the difference in the mean, median, and percentage, as appropriate.

For the remaining analyses, normal, skewed, and categorical variables will be compared using the mean, median, and percentage difference (95\% confidence interval for the difference) and by unpaired t tests, Mann-Whitney, and v2 tests using the sequential rejective Bonferroni procedure of Holm within each analysis to account for multiple testing. For variables that will be logarithmically transformed before analysis, comparisons will be made using the anti-logged differences (interpreted as the ratio of the geometric mean and 95\% confidence interval for the ratio) and Bonferroni corrected $\mathrm{t}$ tests as previously described.

In order to determine whether OSA will be associated with the outcome variables independent of obesity and other known covariates, a regression analysis adjusted for age, BMI, smoking, and alcohol consumption will also be done. Waist circumference, percentage body fat, and fat mass were excluded as covariates because of their high correlation with BMI. Normally distributed, skewed, and log-transformed outcome data would be analysed using multiple linear regression and assumptions would be checked by inspection of the residuals. Categorical outcome data will be analysed using multiple binary logistic regression and assumptions will be checked with the Hosmer-Lemeshow goodness-of-fit test. Statistical analysis will be performed using SPSS version 10 (Chicago, IL, USA).

\section{DISCUSSION}

Running scientific evidences suggest that OSA contributes independently to the rise on cardiometabolic risks, alarming health professionals involved with this subject as for the presence of metabolic dysfunction. During the apneic event hypoxemia associated to hypercapnia occurs, with a consequent stimulation of the sympathetic nervous system causing peripheric vasoconstriction. In addition, a reduction of the negative intrathoracic pressure is observed, as well as a decrease of the cardiac pre- and post-charge. After restoration of the ventilatory flow, there is an increase on the circulating volume (pre-charge) on the validity of the vasoconstriction, leading to repetitive elevations of the arterial pressure (AP) during the night. Chronically, sustained elevations of the AP by stimulation of sympathetic activity can be observed, simultaneously there is decrease on parasympathetic activity, decrease of nitric oxide and release of endothelin. ${ }^{42}$ The hyperaldosteronism can be a cause for resistant hypertension in patients with OSA. Activation of the renin-angiotensin, inflammation, resistance to insulin, decrease on baroreceptors' sensibility, endothelial dysfunction oxidative stress and hyperleptinemia can also be implicated on arterial hypertension's progress. ${ }^{43}$

However, populational longitudinal studies are necessary to prove the causal relation of OSA and metabolic disturbances or the opposite, as well as multi-centric controlled randomized studies to confirm the beneficial effect of therapy with positive pressure on the airways on metabolic disturbs on subjects with OSA. ${ }^{44}$

\section{AUTHOR'S CONTRIBUTION}

All authors contributed equally to this work.

\section{CONFLICTS OF INTEREST}

The author(s) declare that they have no competing interests.

\section{AUTHOR DETAILS}

${ }^{1}$ Faculdade de Ciências Médicas da Santa Casa de São Paulo (SP), Brazil. ${ }^{3}$ Hospital Servidor Público Estadual (HSPE), São Paulo (SP), Brazil.

\section{REFERENCES}

1. Obesity and overweight. Fact sheet. World Health Organization. Updated June 2016 http://www.who.int/mediacentre/factsheets/fs311/en.

2. Knai C, Suhrcke M, Lobstein T. Obesity in Eastern Europe: an overview of its health and economic implications. Econ Hum Biol. 2007 Dec;5(3):392408. 
3. The NHS Information Centre for Health and Social Care. Health Survey for England 2011: Adult trend tables. http://content.digital.nhs.uk/ article/2021/Website-Search productid $=20797 \&$ q=obesity \&sort=Rele vance\&size=10\&page=1\&area=both\#top Publication date: October 31, 2016.

4. Roland Sturm, Aiko Hattori, Morbid Obesity Rates Continue to Rise Rapidly in the US. Int J Obes (Lond). 2013 June ; 37(6): 889-891.

5. Clinical Guidelines on the Identification, Evaluation, and Treatment of Overweight and Obesity in Adults: The Evidence Report: National Institutes of Health. Obes Res. 1998;Suppl 2:51S-209S.

6. Nieto FJ, Young TB, Lind BK, et al.: Association of sleep disordered breathing, sleep apnea and hypertension in a large community-based study: Sleep Heart Health Study. JAMA 2000; 12(283):1829-1836.

7. Drager LF, Bortolotto LA, Krieger EM, Lorenzi-Filho G: Additive Effects of Obstructive Sleep Apnea and Hypertension on Early Markers of Carotid Atherosclerosis. Hipertension. 2008; 53:64-69.

8. Kanaley JA, Goulopoulou SH, Franklin RM, Baynard T, Holmstrup ME, Carhart $\mathrm{R} J \mathrm{r}$, et al.: Plasticity of heart rate signaling and complexity with exercise training in obese individuals with and without type 2 diabetes. In J Obes (Lond)2009; 33(10):1198-206.

9. Prospective Studies Collaboration. Body mass index and cause specific mortality in 900,000 adults: collaborative analyses of 57 prospective studies. Lancet 2009; 373: 1083-96.

10. Grundy SM. Metabolic Syndrome: Connecting and Reconciling Cardiovascular and Diabetes Worlds. JACC 2006, 47, 6:1093-100.

11. Grundy SM, Cleeman JI, Daniels SR, et al. Diagnosis and management of the metabolic syndrome. An American Heart Association/National Heart, Lung, and Blood Institute Scientific Statement. Circulation 2005;112:273552.

12. Park SH, Lindholm B. Definition of metabolic syndrome in peritoneal dialysis. Perit Dial Int 2009;29:S137-44.

13. Reaven GM. Banting Lecture 1988. Role of Insulin Resistance in Human disease. DIABETES, 1988. 37, 1595-1607.

14. Kaur J. A Comprehensive Review on Metabolic Syndrome. Cardiology Research and Practice. 2014, article ID 943162, 21 pages

15. Nestel P, Lyu R, Low LP, Sheu WH, Nitiyanant W, et al. (2007) Metabolic syndrome: recent prevalence in East and Southeast Asian populations. Asia Pac J Clin Nutr 16: 362-7.

16. Eapen D, Kalra GL, Merchant N, Arora A, Khan BV (2009) Metabolic syndrome and cardiovascular disease in South Asians. Vasc Health Risk Manag 5: 731-43.

17. Expert Panel on Detection, Evaluation, and Treatment of High Blood Cholesterol in Adults. Executive Summary of the Third Report of the National Cholesterol Education Program (NCEP) Expert Panel on Detection, Evaluation and Treatment of High Blood Cholesterol in Adults (Adult Treatment Panel III). JAMA 2001;285:2486-97.

18. Drager LF, Lopes HF, Maki-Nunes $C$, et al. The impact of obstructive sleep apneia on metabolic and inflammatory markers in consecutive patients with metabolic syndrome. PLoS One 2010;5:e12065. 3.

19. Trombetta IC, Somers VK, Maki-Nunes C, et al. Consequences of comorbid sleep apneia in the metabolic syndrome implications for cardiovascular risk. Sleep 2010;3:1193-9.

20. Peppard PE, Young T, Palta M, Skatrud J (2000) Prospective study of the association between sleep-disordered breathing and hypertension. $\mathrm{N}$ Engl J Med 342: 1378-1384.

21. Punjabi NM, Sorkin JD, Katzel LI, Goldberg AP, Schwartz AR, et al. (2002) Sleep-disordered breathing and insulin resistance in middle-aged and overweight men. Am J Respir Crit Care Med 165: 677-682.

22. Li J, Thorne LN, Punjabi NM, Sun CK, Schwartz AR, et al. (2005) Intermittent hypoxia induces hyperlipidemia in lean mice. Circ Res 97: 698-706.

23. Savransky V, Jun J, Li J, Nanayakkara A, Fonti S, Moser AB, et al. (2008) Dyslipidemia and atherosclerosis induced by chronic intermittent hypoxia are attenuated by deficiency of stearoyl coenzyme A desaturase. Circ Res 103:1173-1180.
24. Coughlin SR, Mawdsley L, Mugarza JA, Calverley PM, Wilding JP (2004) Obstructive sleep apnoea is independently associated with an increased prevalence of metabolic syndrome. Eur Heart J 25: 735-741.

25. Gruber A, Horwood F, Sithole J, Ali NJ, Idris I. Obstructive sleep apnoea is independently associated with the metabolic syndrome but not insulin resistance state. Cardiovasc Diabetol. 2006, 5: 22.

26. Stephen W, Littleton MD, Babak M: The Pickwickian Syndrome-Obesity Hipoventilation Syndrome. Clin Chest Med. 2009; 30:467-478.

27. Berger KI, Ayappa I, Chart-amontri B, Marfatia A, Sorkin IB, Rapoport DM, et al.: Obesity Hypoventilation Syndrome as a Spectrum of Respiratory Disturbances During Sleep. Chest. 2001; 120(4):1231-1238.

28. Weitzenblum E, Kessler R, Chaouat A: Alveolar hypoventilation in the obese: the obesity-hypoventilation syndrome. Rev. Pneumol. Clin. 2002;58(2):83-90.

29. Sleep-related breathing disorders in adults: recommendations for syndrome definition and measurement techniques in clinical research. The Report of an American Academy of Sleep Medicine Task Force. Sleep. 1999;22:667- 689.

30. Feinsilver SH: Current and future methodology for monitoring sleep. Clinics in Chest Medicine. 1998;19(1):213-18.

31. Wilcox I, McNamara SG, Collins FL, Grunstein RR, Sullivan CE. "Syndrome $Z^{\prime \prime}$ : the interaction of sleep apnoea, vascular risk factors and heart disease. Thorax 1998;53(Suppl 3):S25-28.

32. World Health Organization. Obesity: preventing and managing the global epidemic. Report of a World Health Organization Consultation. Geneva: World Health Organization, 2000. p. 256. WHO Obesity Technical Report Series, n. 284.

33. Gabrielsen, AM, Lund MB, Kongerud J, Viken, KE, Roislien J, Hjelmesaeth $J$. The relationship between anthropometric measures, blood gases, and lung function in morbidly obese white subjects. Obes Surg. 2011, 21, 4: 485-491.

34. Hingorjo MR, Qureshi MA, Mehdi A. Neck circumference as a useful marker of obesity: A comparison with body mass index and waist circumference. J Pak Med Assoc 2012;62(1):36-40.

35. Vallianou NG, Evangelopoulos AA, Bountziouka V, et al. Neck circumference is correlated with triglycerides and inversely related with HDL cholesterol beyond BMI and waist circumference. Diabetes Metab Res Rev 2013;29:90-97.

36. Murray WJ: A new method for measuring daytime sleepiness: the Epworth sleepiness scale. Sleep. 1991;14(6):540-545.

37. Murray WJ: Reliability and factor analysis of Epowoth sleepiness scale. Sleep. 1992; 15(4):376-81.

38. Netzer NC, Stoohs RA, Netzer CM, et al. Using the Berlin questionnaire to identify patients at risk for the sleep apnea syndrome. Ann Intern Med 1999;131(7):485-491.

39. Rechtschaffen A, Kales A: A manual of standardized terminology: techniques and scoring system for sleep stages of human subjects. Los Angeles: Brain Information Service/Brain Research Institute; 1968. Psychiatry and Clinical Neurosciences. 2001; 55:305-310.

40. Iber C, Ancoli-Israel S, Chesson Jr A, Quan S: The AASM manual for the scoring of sleep and associated events: rules, terminology and technical specifications. Westchester: merican Academy of Sleep Medicine; 2007.

41. American Academy of Sleep Medicine. The AASM Manual for the scoring of sleep and associated events. Rules, terminology and technical especifications; 2007

42. Goodfriend TL, Calhoun DA. Resistant hypertension, obesity, sleep apnea, and aldosterone: theory and therapy. Hypertension 2004;43:518-24.

43. Peppard PE, Young T, Palta M, Skatrud J. Prospective study of the association between sleep-disordered breathing and hypertension. $\mathrm{N}$ Engl J Med 2000;342:1378-84

44. Marin JM, Carrizo SJ, Vicente E, Agusti AGN. Long-term cardiovascular outcomes in men with obstructive sleep apnoea hypopnoea with or without treatment with continuous positive airway pressure: an observational study. Lancet 2005;365:1046-53 